\title{
Sistema de Aseguramiento de Calidad (SAC), basado en la metodología Six Sigma para reducir la variabilidad de tono en los procesos de inyección-soplo de plástico
}

\section{Quality Assurance System (SAC), based on the Six Sigma methodology to reduce tone variability in plastic injection-blow processes}

\author{
SOTO-LEYVA, Yasmin †*, CARMONA-VELÁZQUEZ, Araceli, LIEVANO-MORENO, Claudia \\ Patricia y AHUACATITLA-PÉREZ, José Miguel
}

Tecnológico Nacional de México/Instituto Tecnológico Superior de Huauchinango, México.
Universidad Xicotepetl, A.C., México.

ID $1^{\text {er }}$ Autor: Yasmin, Soto-Leyva / ORC ID: 0000-0003-2652-7065, CVU CONACYT ID: 951464

ID $1^{\text {er }}$ Coautor: Araceli, Carmona-Velázquez / ORC ID: 0000-0002-4334-0262, CVU CONACYT ID: 1062056

ID $2^{\text {do }}$ Coautor: Claudia Patricia, Liévano-Moreno / ORC ID: 0000-0003-4407-0724, CVU CONACYT ID: 209534

ID $3^{\text {er }}$ Coautor: José Miguel, Ahuacatitla-Pérez / ORC ID: 0000-0001-5336-8966, CVU CONACYT ID: 951466

DOI: $10.35429 /$ JTO.2020.14.4.22.31

Recibido 20 de Julio, 2020, Aceptado, 30 de Diciembre, 2020

\begin{abstract}
Resumen
La presente aplicación desarrolla un Sistema de Aseguramiento de la Calidad (SAC), diseñado conforme a los requerimientos del proceso de producción de inyección-soplo correspondiente a la industria del plástico la cual aporta el $2.6 \%$ del PIB nacional. El objetivo plantea el mejoramiento cuantitativo del nivel Sigma de la empresa analizada, buscando una reducción del $20 \%$ en los costos generados por el problema denominado "variación de tono", originado en la línea productiva de inyección-soplo de plástico, basándose en la metodología Six Sigma (DMAIC). La metodología está constituida por cinco fases, mismas que permiten el logro del objetivo planteado: Fase 1. Definir problema, Fase 2. Recopilación y análisis de datos, Fase 3. Análisis de las causas y factores críticos del problema, Fase 4. Propuesta de mejora de implementación de herramientas para solucionar el problema, Fase 5. Controlar: diseño de un sistema de medición para asegurar la estabilidad de la calidad del producto. Con la implementación de las herramientas derivadas del análisis y de los resultados obtenidos en cada fase, se obtiene un nivel de $3.7 \sigma$ incrementando en $0.3 \sigma$, con respecto al nivel Sigma inicial de 3.4, traducido en un ahorro económico anual de $\$ 141,120.00$.
\end{abstract}

Six Sigma, Variación, SAC

\begin{abstract}
This application develops a Quality Assurance System (SAC), designed in accordance with the requirements of the injection-blow production process corresponding to the plastics industry, which contributes $2.6 \%$ of the national GDP. The objective raises the quantitative improvement of the Sigma level of the analyzed company, seeking a reduction of $20 \%$ in the costs generated by the problem called "tone variation", originated in the plastic injection-blow production line, based on the methodology Six Sigma (DMAIC). The methodology consists of five phases, which allow the achievement of the proposed objective: Phase 1. Define the problem, Phase 2. Data collection and analysis, Phase 3. Analysis of the causes and critical factors of the problem, Phase 4. Proposal for improvement of the implementation of tools to solve the problem, Phase 5. Control: design of a measurement system to ensure the stability of product quality. With the implementation of the tools derived from the analysis and the results obtained in each phase, a level of $3.7 \sigma$ is obtained, increasing by $0.3 \sigma$, with respect to the initial Sigma level of 3.4 , translated into an annual economic saving of $\$$ $141,120.00$.
\end{abstract}

Six Sigma, Variation, SAC

Citación: SOTO-LEYVA, Yasmin, CARMONA-VELÁZQUEZ, Araceli, LIEVANO-MORENO, Claudia Patricia y AHUACATITLA-PÉREZ, José Miguel. Sistema de Aseguramiento de Calidad (SAC), basado en la metodología Six Sigma para reducir la variabilidad de tono en los procesos de inyección-soplo de plástico. Revista de Operaciones Tecnológicas. 2020. 4-14:22-31.

\footnotetext{
*Correspondencia al Autor (Correo Electrónico: yasmin.soto@ huauchinango.tecnm.mx)

$\dagger$ Investigador contribuyendo como primer autor.
} 


\section{Introducción}

Con el paso del tiempo las empresas productoras de plástico se han posicionado con mayor auge en el desarrollo económico del país, esto trae consigo que las organizaciones mejoren significativamente la productividad y la calidad de los productos, mediante la implementación de herramientas que permitan identificar y eliminar los principales problemas provenientes de la calidad de los productos que procesan (defectos) considerados responsabilidad del fabricante (Josep Solé Feliu,1996), los cuales generan altos costos de reprocesos y perdidas inmediatas de clientes potenciales.

La presente aplicación se efectuó en una empresa que fabrica diferentes tipos de envases de plástico, teniendo como principales clientes a la industria farmacéutica (ampolletas inyectables), alimenticia (recipientes, vasos, tapas) y cosméticos (estuches, depósitos, jaboneras), en el periodo comprendido de enero-julio 2019 la organización recibió un promedio mensual de 32 reclamos/inconformidades que originaron rechazos de lotes de producción, y reprocesos de los productos, ocasionando una disminución del $10 \%$ de los clientes que conforman la red de compra de los principales envases, debido a los defectos de variación de tono que presentan los productos representando una pérdida anual de 1.3 millones de pesos.

El proceso de pigmentación que permite asignar el tono a los envases se efectúa en el área de inyección-soplo, que está conformado por una mesa rotativa, que gira en pasos de 80140 grados (Figura 1 Mesa rotativa "Proceso de pigmentación"). En la cara horizontal de la mesa se instalan 5 agujas especiales o varillas centrales (core rods), donde se fabrica la preforma que más tarde es soplada y pigmentada para formar el envase terminado.

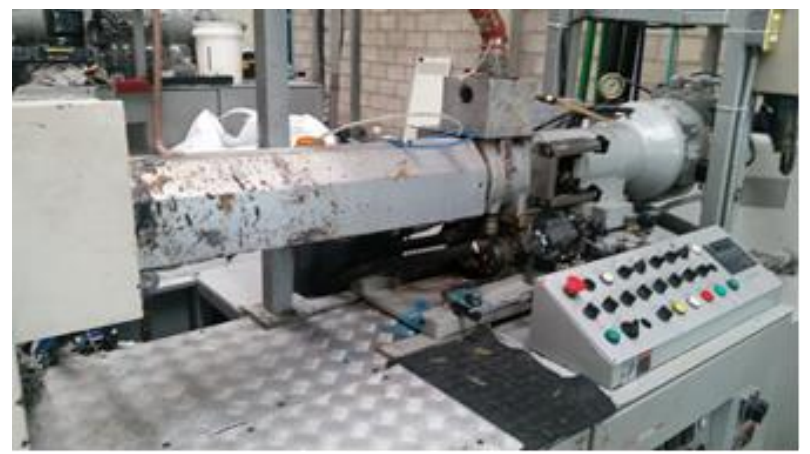

Figura 1 Mesa rotativa "Proceso de pigmentación" Fuente: Elaboración propia con información de la empresa (2020)

La problemática de variación de tono se presenta por diversas causas supuestas: falta de homogeneización del concentrado sobre la resina, ciclo excesivamente rápido, material degradándose, exceso de pigmentos, falta de resistencia térmica del concentrado, etc. Para identificar y comprobar la causa raíz del problema se aplica una ACR (Análisis de Causa Raíz), posteriormente se aplican herramientas de Ingeniería para disminuir el nivel de variabilidad de tono que se presenta en los lotes de producto terminado $(2.9 \%$ de las piezas finales), medido al inicio del proyecto en agosto 2019, contemplando que la empresa maneja un estándar máximo de productos (envases) con variabilidad de tono del $1.1 \%$.

La búsqueda de la metodología idónea para el aseguramiento de la calidad que controle y mejore el proceso operativo, para aumentar notablemente el nivel Sigma, reducir los costos de reprocesamiento y disminuir los defectos generados por variación de tono, en el proceso de inyección-soplo de plásticos para la fabricación de envases trae consigo la selección e implementación de la metodología Six Sigma que tiene sus orígenes en la necesidad de mejorar y solucionar problemas complejos. $\mathrm{Su}$ creador fue el doctor Mikel Harry, que la desarrolló como una herramienta de control y disminución de la variación en los procesos complejos. Six Sigma, según López (2011), es un enfoque revolucionario de gestión que mide y mejora la calidad. Ha llegado a ser un método de referencia para, al mismo tiempo, satisfacer las necesidades de los clientes y lograrlo con niveles próximos a la perfección. 
Puede ser analizada desde dos puntos de vista: estadístico y estratégico, en el primero, Six Sigma significa "seis desviaciones estándar de la media", lo cual se traduce matemáticamente a menos 3.4 defectos por millón, mientras que, desde el segundo punto de vista, Six Sigma ajusta a los procesos con la mínima tolerancia de defectos posibles como una forma de reducir los desperdicios y las irregularidades tanto en los productos como en los servicios.

En el corazón de la metodología Six Sigma se encuentra el modelo DMAIC para los procesos existentes que caen por debajo de las especificaciones $y$ buscan una mejora incremental (Bikram, 2015). Este consiste en la aplicación de un proceso estructurado en cinco fases: definir, medir, analizar, mejorar, controlar (Pérez, 2014).

El aseguramiento de la calidad, o garantía de calidad, es, según la norma ISO 8402, el conjunto de acciones planificadas y sistemáticas necesarias para proporcionar la confianza adecuada de que un producto $\mathrm{o}$ servicio satisfará los requerimientos relativos a la calidad. Asimismo, según la familia de ISO 9000 el objetivo principal del SAC es garantizar la permanencia en el tiempo de los logros obtenidos.

Tomando en cuenta lo anterior el presente estudio tiene por objetivo desarrollar un Sistema de Aseguramiento de Calidad (SAC) con base en la metodología DMAIC correspondiente a Six Sigma, para el proceso de inyección-soplo de plástico, aplicando herramientas cuantitativas y cualitativas para la eliminación de las fuentes que ocasionan la variabilidad, enfocándonos al cumplimiento de la característica principal que define a los envases producidos: tonalidad.

\section{Metodología por desarrollar}

A continuación, se presentan las fases correspondientes a la metodología:

\section{Fase 1. Definir problema}

La pérdida de clientes potenciales trajo consigo la aplicación de un análisis estadístico para determinar cuáles son los principales problemas que ocasionan el rechazo de los lotes de producción, las etapas que constituyen el estudio son:

Etapa 1. Feed Back Clientes (análisis de reclamos/quejas).

- Etapa 2. Defectos presentados en área inyección-soplo.

Etapa 1. En primera instancia se realizó un análisis del total de los reclamos/quejas presentadas para conocer los factores que ocasionaban la pérdida de los clientes potenciales, las variables analizadas son: calidad, costo, tiempo de entrega (Gráfico 1 Feed Back Clientes (análisis de reclamos/quejas)).

Feed Back Clientes (análisis de reclamos/quejas)

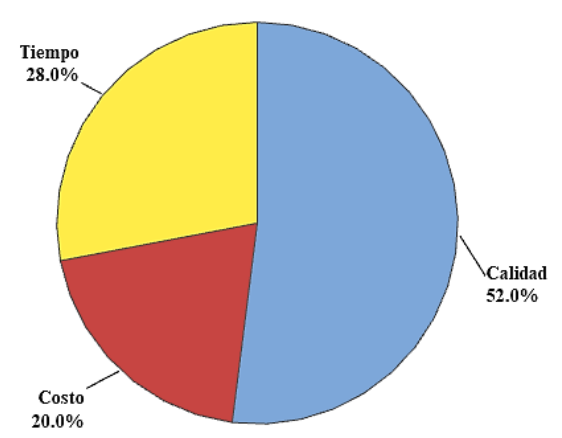

Gráfico 1 Feed Back Clientes (análisis de reclamos/quejas)

Fuente: Elaboración propia con información de la empresa (2020)

El estadístico histórico indica que las inconformidades presentan un porcentual de: calidad $52 \%$, costo $20 \%$, tiempo de entrega $28 \%$ siendo la calidad la fuente que origina en mayor grado la insatisfacción de los clientes originando la reducción de la cartera de clientes prevista para el año 2020.

Etapa 2. Se procedió a determinar el principal problema de calidad (defectos) que ocasiona los rechazos de lotes de producción de variabilidad que se presentó en los lotes de producción rechazados. 
Se realizó un diagrama de pareto por turno, para identificar la frecuencia con la que se presentan cada uno de los defectos, (Gráfico 2 Análisis de defectos en el primer turno, Gráfico 3 Análisis de defectos en el segundo turno, Gráfico 4 Análisis de defectos en el tercer turno). De manera general los defectos presentados son: variación de tono, variación de espesores, hebra, delaminación, rechupe, punto desviado y rebaba, tomando la información de los 3 diferentes turnos laborales.

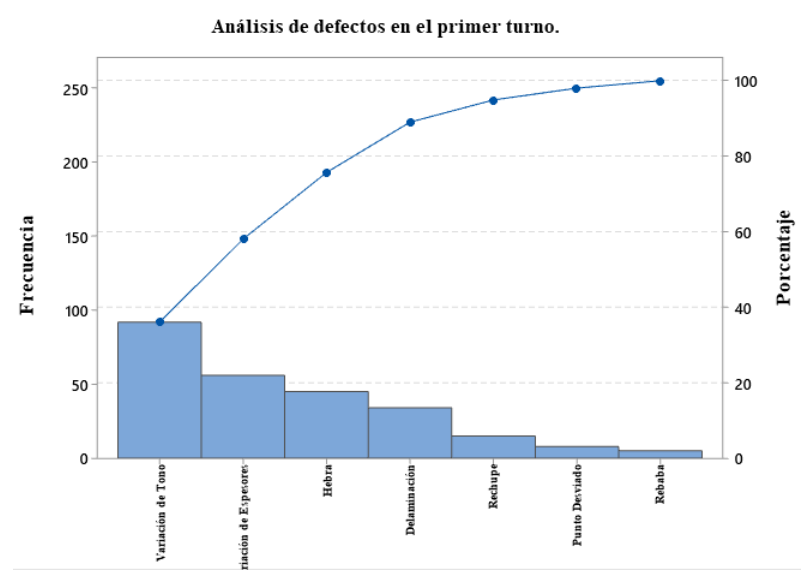

Gráfico 2 Análisis de defectos en el primer turno Fuente: Elaboración propia con información de la empresa (2020)

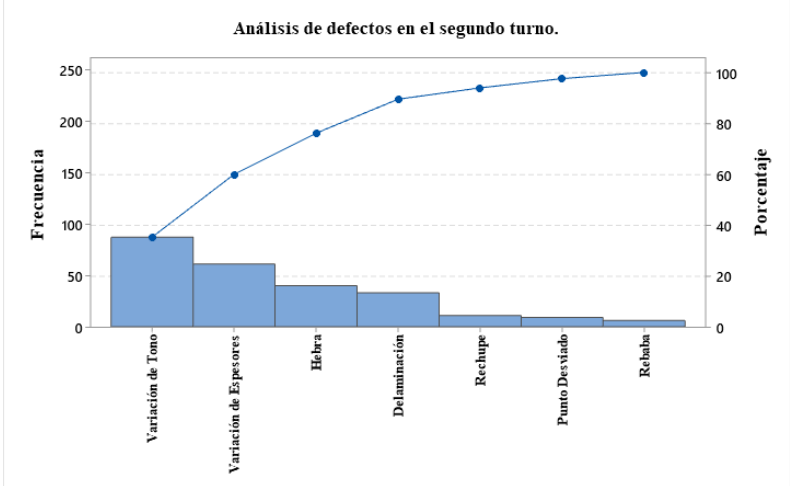

Gráfico 3 Análisis de defectos segundo turno

Fuente: Elaboración propia con información de la empresa (2020)

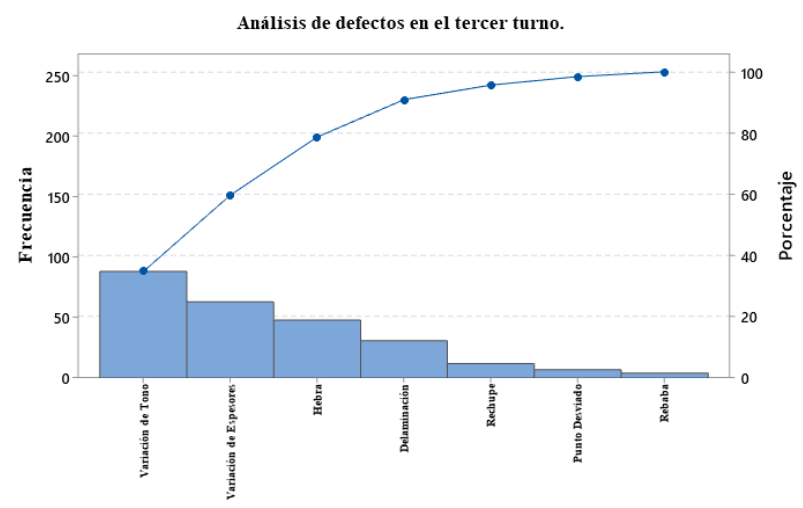

Gráfico 4 Análisis de defectos en el tercer turno

Fuente: Elaboración propia con información de la empresa (2020)

ISSN: 2523-6806

ECORFAN ${ }^{\circledR}$ Todos los derechos reservados
Analizando los Gráficos 1, 2 y 3 observamos que el tipo de defecto que se presenta con mayor frecuencia es la variación de tono para el primer turno $36 \%$ para el segundo turno $35 \%$ y para el tercer turno $35 \%$ por lo que se aplicaran acciones correctivas a este tipo de defecto en primera instancia.

\section{Fase 2. Recopilación y análisis de datos}

El proceso de asignación de tonos se realiza en la zona de pigmentación que se encuentra ubicada en el área de inyección-soplo, contabilizando 10 máquinas dedicadas a esta operación (Figura 2 Máquinas para procesos de pigmentación), el proceso de asignación de tono se realiza mediante la fundición de pigmentos y colorantes en el cañón a temperaturas mayores a $210^{\circ}$, el material fundido es transportado por el husillo para ser inyectado en el centro de la preforma, la dosificación de la pigmentación debe ser precisa, previamente el molde preforma se apertura y es trasferido a la siguiente estación.

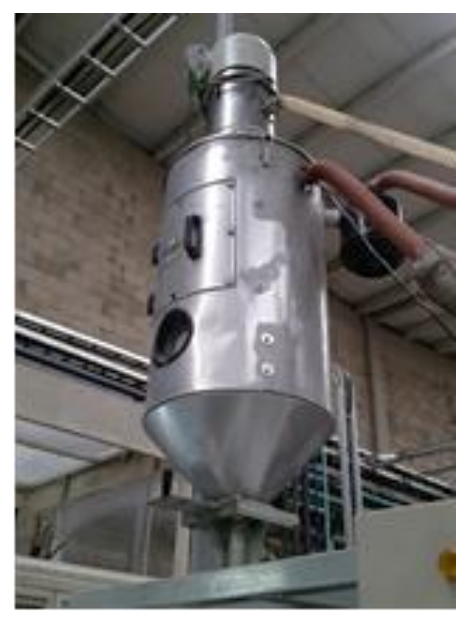

Figura 2 Máquinas para procesos de pigmentación Fuente: Elaboración propia con información de la empresa (2020)

La metodología de análisis se efectuó aplicando un muestreo aleatorio a los envases procesados en las distintas máquinas y en horarios diversos, mediante el cual se determinaron las máquinas que ocasionaban mayor cantidad de defectos por variación de tono, los resultados obtenidos se muestran a continuación (Tabla 1 Control general de defectos por máquina). 


\begin{tabular}{|l|r|r|}
\hline \multicolumn{1}{|c}{ Máquina } & \multicolumn{1}{c}{$\begin{array}{c}\text { Frecuencia de } \\
\text { presentación } \\
\text { defectos }\end{array}$} & $\begin{array}{c}\text { \% } \\
\text { Variación } \\
\text { de tono }\end{array}$ \\
\hline R-01 & 190 & $42.37 \%$ \\
\hline R-02 & 82 & $23.10 \%$ \\
\hline R-03 & 151 & $32.85 \%$ \\
\hline R-04 & 150 & $32.72 \%$ \\
\hline R-05 & 125 & $29.38 \%$ \\
\hline R-06 & 195 & $45.57 \%$ \\
\hline R-07 & 192 & $43.43 \%$ \\
\hline R-08 & 148 & $31.13 \%$ \\
\hline R-09 & 148 & $31.13 \%$ \\
\hline R-10 & 124 & $29.00 \%$ \\
\hline Promedio general & 151 & $34.06 \%$ \\
\hline $\begin{array}{l}\text { Promedio Máquinas } \\
\text { con } \\
\text { incidencia. }\end{array}$ & 192 & $43.79 \%$ \\
\hline
\end{tabular}

Tabla 1 Control general de defectos por máquina Fuente: Elaboración propia con información de la empresa (2020)

Se detecta que las máquinas que presentan mayor concentración de defectos por variación de tono son $\mathrm{R}-01=42.37 \%$, R$06=45.57 \%$, R- $07=43.43 \%$, con un promedio de 192 defectos mensuales.

La frecuencia de presentación de defectos mensuales genera una proporción del $2.9 \%$ de piezas terminadas con defecto por variación de color y un correlacional de piezas de calidad del $97.1 \%$ (Gráfico 5 Análisis correlacional inicial de piezas producidas " $\%$ piezas defectuosas, \%piezas de calidad").

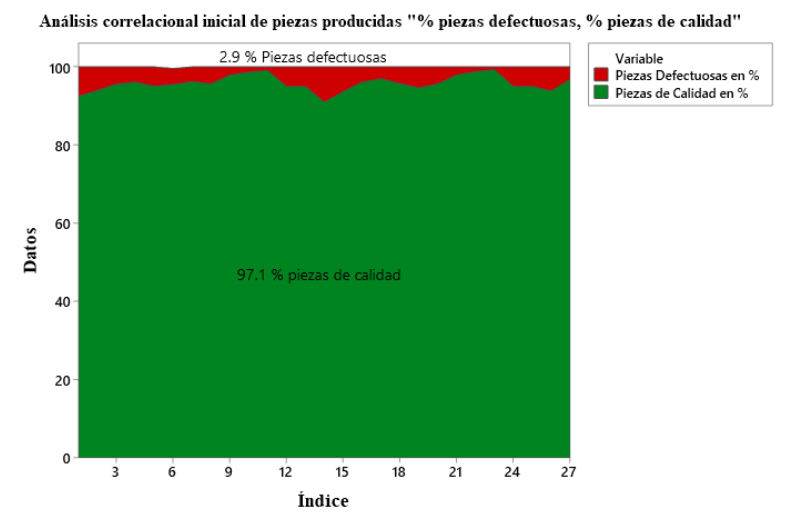

Gráfico 5 Análisis correlacional inicial de piezas producidas "\% piezas defectuosas, \%piezas de calidad" Fuente: Elaboración propia con información de la empresa (2020)

Esta proporción nos muestra que el valor Sigma actual es de $3.4 \sigma$, siendo un nivel por debajo de la calidad "normal" conforme al nivel Sigma promedio en las industrias productoras de plástico (Tabla 2 Abstracto de niveles de asignación Sigma).

\begin{tabular}{|c|c|c|}
\hline Sigma & $\begin{array}{c}\text { \% Unidades de } \\
\text { Calidad }\end{array}$ & $\begin{array}{l}\text { \% Unidades } \\
\text { defectuosas }\end{array}$ \\
\hline 4.0 & $99.38 \%$ & $0.62 \%$ \\
\hline 3.9 & $99.18 \%$ & $0.82 \%$ \\
\hline 3.8 & $98.9 \%$ & $1.1 \%$ \\
\hline 3.7 & $98.6 \%$ & $1.4 \%$ \\
\hline 3.6 & $98.2 \%$ & $1.8 \%$ \\
\hline 3.5 & $97.7 \%$ & $2.3 \%$ \\
\hline 3.4 & $97.1 \%$ & $2.9 \%$ \\
\hline 3.3 & $96.4 \%$ & $3.6 \%$ \\
\hline 3.2 & $95.5 \%$ & $4.5 \%$ \\
\hline 3.1 & $94.4 \%$ & $5.6 \%$ \\
\hline 3.0 & $93.3 \%$ & $6.7 \%$ \\
\hline
\end{tabular}

Tabla 2 Abstracto de niveles de asignación Sigma Fuente: bookdown.org/asun_mayoral/book-l6s/dmaic2medir.html (2020)

\section{Fase 3. Análisis de las causas y factores críticos del problema}

Para el análisis de causas del problema se hace uso de la metodología ACR (Análisis de Causa Raíz), mediante la cual se identificó de forma reactiva las causas que originaban la variabilidad de tono.

Para la realización del estudio se analizan las máquinas que presentaron mayor nivel de incidencia R-01, R-06, R-07, los resultados se plasman en un Diagrama de Ishikawa (Figura 3 Diagrama de Ishikawa: Análisis de Causa Raíz).

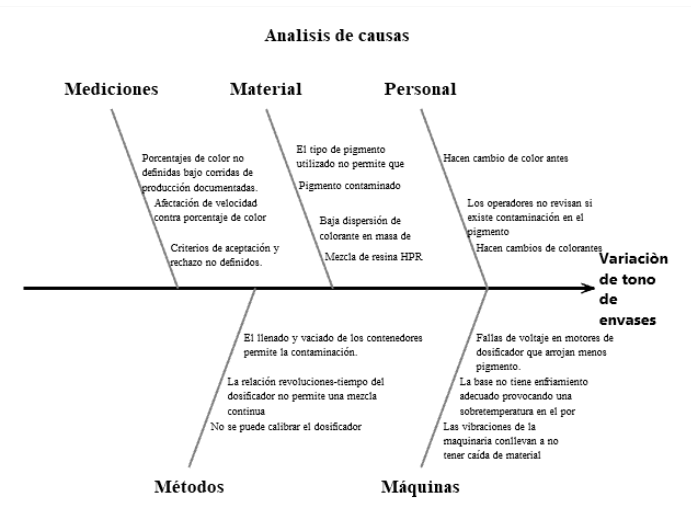

Figura 3 Diagrama de Ishikawa: Análisis de Causa Raíz Fuente: Elaboración propia con información de la empresa (2020)

Derivado de los resultados del ACR, se realizó un estudio de las causas de mayor relevancia a través de la herramienta de los 5 ¿Por qué?’s (Tabla 3 Diagrama de los 5 ¿Por qué?'s). 


\begin{tabular}{|c|c|c|c|c|}
\hline \multicolumn{2}{|c|}{ Causa } & & $2^{\circ}$ & \\
\hline & & $\begin{array}{l}\text { Contaminación } \\
\text { de cubetas de } \\
\text { pigmento con } \\
\text { diferentes } \\
\text { colores }\end{array}$ & $\begin{array}{c}\text { Sobre } \\
\text { ajustes a } \\
\text { porcentajes } \\
\text { de color }\end{array}$ & $\begin{array}{c}\text { Criterios } \\
\text { de } \\
\text { aceptación } \\
\text { o rechazo }\end{array}$ \\
\hline $1^{\circ}$ & $\begin{array}{l}\text { ¿Por } \\
\text { qué? }\end{array}$ & $\begin{array}{lr}\text { Los } & \text { operadores } \\
\text { no } & \text { "sopletean" } \\
\text { de } & \text { forma } \\
\text { correcta } & \text { ni } \\
\text { previenen } & \\
\text { contaminación. }\end{array}$ & $\begin{array}{l}\text { Los cambios } \\
\text { de lote de } \\
\text { pigmento } \\
\text { provocan } \\
\text { variaciones } \\
\text { de color. }\end{array}$ & $\begin{array}{l}\text { El criterio } \\
\text { cambia } \\
\text { según la } \\
\text { persona } \\
\text { que lo } \\
\text { audita. }\end{array}$ \\
\hline $2^{\circ}$ & $\begin{array}{l}\text { ¿Por } \\
\text { qué? }\end{array}$ & $\begin{array}{lr}\text { No } & \text { son } \\
\text { conscientes } & \text { del } \\
\text { grado } & \text { de } \\
\text { afectación. } & \end{array}$ & \begin{tabular}{l}
\multicolumn{2}{c}{ Se } \\
mezclan los \\
lotes de \\
pigmento.
\end{tabular} & $\begin{array}{l}\text { No están } \\
\text { establecido } \\
\text { s dos } \\
\text { límites de } \\
\text { aceptación }\end{array}$ \\
\hline $3^{\circ}$ & $\begin{array}{l}\text { ¿Por } \\
\text { qué? }\end{array}$ & $\begin{array}{l}\text { No se sabe } \\
\text { cuántas } \\
\text { variaciones de } \\
\text { color produce la } \\
\text { contaminación. }\end{array}$ & $\begin{array}{l}\text { No se tiene } \\
\text { control de } \\
\text { los } \\
\text { diferentes } \\
\text { lotes que } \\
\text { llegan. }\end{array}$ & $\begin{array}{l}\text { La } \\
\text { medición } \\
\text { es visual. }\end{array}$ \\
\hline $4^{\circ}$ & $\begin{array}{l}\text { ¿Por } \\
\text { qué? }\end{array}$ & $\begin{array}{lr}\text { No hay un } \\
\text { responsable } \\
\text { cargo. }\end{array}$ & $\begin{array}{l}\text { No se } \\
\text { registran las } \\
\text { entradas de } \\
\text { los lotes en } \\
\text { el almacén. }\end{array}$ & $\begin{array}{l}\text { No hay un } \\
\text { equipo de } \\
\text { medición } \\
\text { mejor. }\end{array}$ \\
\hline $5^{\circ}$ & $\begin{array}{l}\text { ¿Por } \\
\text { qué? }\end{array}$ & $\begin{array}{lr}\text { No hay } & \text { un } \\
\text { método } & \text { que } \\
\text { corrija a los } \\
\text { operadores } & \text { para } \\
\text { tener } & \text { su } \\
\text { pigmento } & \\
\text { limpio. } & \\
& \end{array}$ & $\begin{array}{l}\text { No hay un } \\
\text { método } \\
\text { definido. }\end{array}$ & $\begin{array}{l}\text { No se ha } \\
\text { cotizado } \\
\text { ningún } \\
\text { equipo de } \\
\text { medición. }\end{array}$ \\
\hline
\end{tabular}

Tabla 3 Diagrama de los 5 ¿Por qué?'s

Fuente: Elaboración propia con información de la empresa (2020)

La aplicación de la herramienta antes descrita permitió detectar las fuentes de origen del problema de variación de tono: contaminación, diferencia entre lotes de pigmentos, dosificación, homogenización.

\section{Fase 4. Propuesta de mejora implementación de herramientas para solucionar el problema}

El proceso de mejora incluye la aplicación de las herramientas siguientes: (Figura 4 Herramientas de mejoras implementadas).

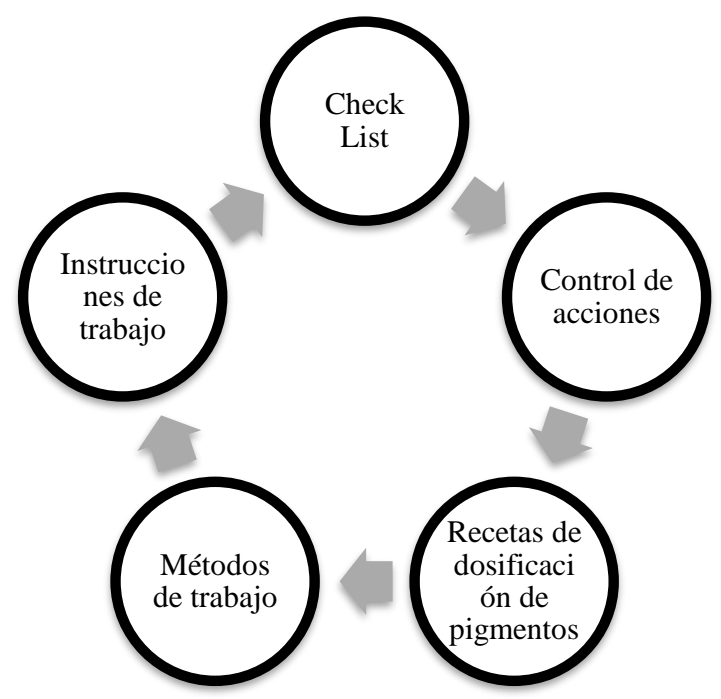

Figura 4 Herramientas de mejoras implementadas Fuente: Elaboración propia (2020)

La primera mejora está orientada a la eliminación de la diferencia entre los lotes de pigmentación: se instituyó un check list mediante el cual se corrige y previene la variación de color por contaminación de lotes de pigmentación, buscando reducir las quejas internas y externas que se generan por variación de tono, este check list deberá de ser llenado por los operadores al final de cada turno y así el operador siguiente estará enterado de las condiciones operativas del turno anterior, para poder continuar trabajando de una manera adecuada (Ver anexo 1; Figura 5 Check List).

Los procesos de contaminación que se presentan son reducidos con el uso de un control de acciones correctivas, preventivas y de mejora que permiten el aseguramiento de la calidad del producto, se mide en periodos de 27 días, durante los 3 turnos, teniendo como objetivo disminuir los niveles de contaminación que generan piezas con defectos de variación de tono en las máquinas R-01, R-06, R-07 (Anexo 2; Figura 6 Control de acciones).

La tercera mejora consiste en el diseño de recetas de dosificación de pigmentos/colorantes y establecimiento de políticas de homogenización para el desarrollo de las actividades operativas a través de la creación de métodos de trabajo e instrucciones de trabajo. 
Fase 5. Controlar: diseño de un sistema de medición para asegurar la estabilidad de la calidad del producto

El método de aseguramiento se aplicó a través de la generación de un certificado de calidad que avala que los productos de cada lote cumplen con las especificaciones y características que el cliente requiere (puntos máximos y mínimos).

El certificado de calidad está diseñado conforme a los requerimientos de cada cliente contemplando el giro comercial al que pertenecen.

\section{Resultados}

Las distintas fases permitieron obtener los siguientes resultados:

La Fase 1 denotó las principales causas que ocasionaban la disminución de los clientes potenciales, visualizando cuales son los defectos más frecuentes que ocasionan el rechazo de los lotes ya fabricados, considerando que por variación de tono se tiene en promedio el $35.33 \%$ de las pérdidas anuales.

La Fase 2 determinó las máquinas de inyección-soplo que generaban mayor cantidad de defectos, el análisis cualitativo proyectó un estudio asertivo determinando que las maquinas $\mathrm{R}-01=42.37 \%, \mathrm{R}-06=45.57 \%$, R-07 $=43.43 \%$ exponen mayores probabilidades de fabricar piezas con variedad de tono, propiciando el análisis de las fichas técnicas y el proceso operativo que se lleva a cabo en cada una de ellas para con esto determinar las fuentes que originan el problema.

El proceso de recolección y análisis de datos trajo consigo el cálculo del nivel Sigma que se presentó al inicio del proyecto conforme al \% de productos defectuosos generados a partir de la variación de tono:

I. $\quad 2.9 \%$ de piezas defectuosas; $97.1 \%$ piezas de calidad $=3.4 \sigma$ (Tabla 2 . Abstracto de niveles de asignación Sigma).
Las propuestas generadas en la Fase 3, disminuyeron notablemente las fuentes de origen que ocasionaban piezas defectuosas, la creación de metodologías e instrucciones de trabajo para los operarios del área de inyecciónsoplo estandarizaron las actividades que se realizaban como parte del proceso de fabricación. La aplicación del check list redujo la presentación de quejas/inconformidades de manera externa, de un conteo de $32 \mathrm{al}$ inicio de la aplicación se presentaron solo 7 al término del proyecto (Gráfico 6 Análisis de quejas/inconformidades de los clientes potenciales).

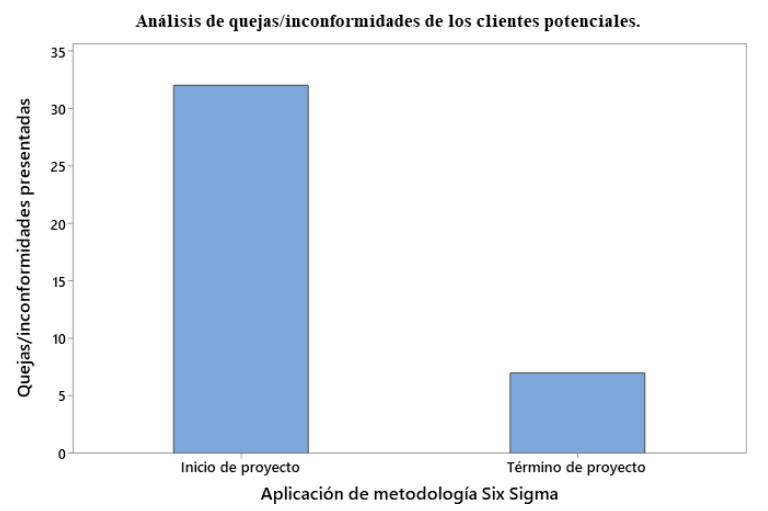

Gráfico 6 Análisis de quejas/inconformidades de los clientes potenciales

Fuente: Elaboración propia con información de la empresa (2020)

Una vez aplicadas las herramientas de mejora se asignó un ciclo de validación de 27 días en los 3 turnos laborales, monitoreando la cantidad de defectos por variación de color originados en las maquinas R-01, R-06, R-07 (Tabla 4 Control de defectos de máquinas R-01, R-06, R-07; ciclo de validación).

\begin{tabular}{|l|l|r|}
\hline Máquina & \multicolumn{1}{c}{$\begin{array}{c}\text { Frecuencia de } \\
\text { presentación de } \\
\text { defectos }\end{array}$} & $\begin{array}{c}\text { \% Variación } \\
\text { de tono }\end{array}$ \\
\hline R-01 & 117 & $26.30 \%$ \\
\hline R-06 & 114 & $25.42 \%$ \\
\hline R-07 & 112 & $24.97 \%$ \\
\hline Promedio & 115 & $25.56 \%$ \\
\hline
\end{tabular}

Tabla 4 Control de defectos de máquinas R-01, R-06, R07; ciclo de validación

Fuente: Elaboración propia con información de la empresa (2020) 
En promedio contabilizamos 115 defectos por variación de tono en los ciclos de validación que se monitorearon, es decir que se registró más de 1 variación de color por turno en las máquinas R-01, R-06, R-07, mostrando una disminución del $33 \%$ de incidencias con respecto a los 192 defectos promedio registradas al inicio del proyecto (Tabla 1 Control general de defectos por máquina).

La proporción correspondiente de piezas defectuosas por variación de color fue de $1.40 \%$, lo que significa una reducción de $1.5 \%$ en el porcentaje de defectos contabilizados de manera inicial; con un correlacional de $98.60 \%$ piezas de calidad (Gráfico 7 Análisis correlacional final de piezas producidas "\% piezas defectuosas, \% Piezas de calidad”).

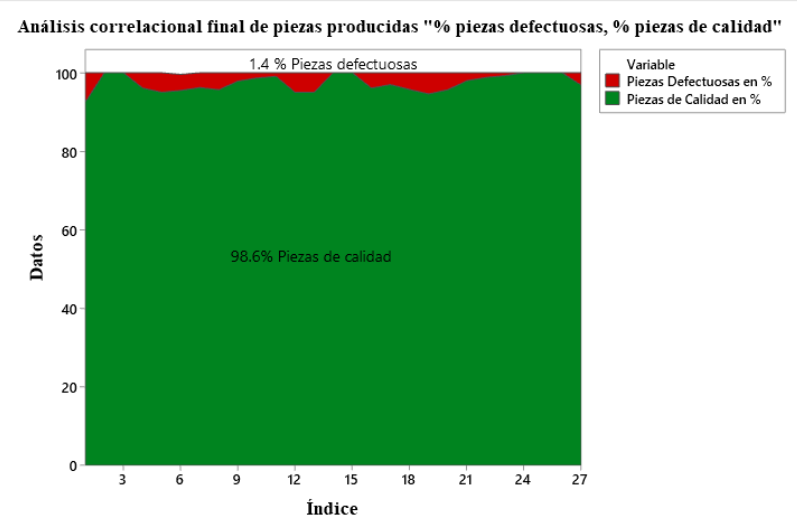

Gráfico 7 Análisis correlacional final de piezas producidas "\% piezas defectuosas, \% Piezas de calidad" Fuente: Elaboración propia con información de la empresa (2020)

Esta proporción nos muestra entonces que el nivel Sigma actual es de $3.7 \sigma$ (Tabla 3 Abstracto de niveles de asignación Sigma).

\section{Anexos}

\section{Anexo 1}

\begin{tabular}{c|l|l|}
\hline CHECK LIST DE & FECHA & \\
ENTREGA Y RECEPCIÓN & TURNO & \\
DEL TURNO DE LOS & OPERADOR & \\
OPERADORES & QUE & \\
& ENTREGA & \\
\cline { 2 - 3 } & OPERADOR & \\
& QUE RECIBE & \\
\hline \multirow{3}{*}{ ACTIVIDADES DEL OPERADOR OUE ENTREGA EL TURNO }
\end{tabular}

\begin{tabular}{|l|l|l|l|}
\hline Actividades & Si & No & Observaciones \\
\hline $\begin{array}{l}\text { Se realizó de manera correcta el llenado de la } \\
\text { bitácora de producción. }\end{array}$ & & & \\
\hline $\begin{array}{l}\text { Se encuentra limpia y ordenada el área de } \\
\text { trabajo. }\end{array}$ & & & \\
\hline $\begin{array}{l}\text { Se realizaron cambios de molde o de orden de } \\
\text { producción. }\end{array}$ & & & \\
\hline $\begin{array}{l}\text { Se llenaron correctamente los formatos de } \\
\text { parámetros de moldeo. }\end{array}$ & & & \\
\hline $\begin{array}{l}\text { Se pesó la preforma y la plasta resultante de } \\
\text { las distintas máquinas. }\end{array}$ & & & \\
\hline $\begin{array}{l}\text { Se llenaron correctamente los formatos de } \\
\text { avisos de falla. }\end{array}$ & & & \\
\hline $\begin{array}{l}\text { Se modificaron los parámetros de moldeo } \\
\text { durante el turno. }\end{array}$ & & & \\
\hline $\begin{array}{l}\text { Se monitoreo cada una de las maquinas que } \\
\text { estuvieron trabajando. }\end{array}$ & & & \\
\hline $\begin{array}{l}\text { Se inspeccionó que el pigmento no estuviera } \\
\text { contaminado. }\end{array}$ & & & \\
\hline $\begin{array}{l}\text { Se inspeccionó que el pigmento líquido no } \\
\text { estuviera asentado en el fondo. }\end{array}$ & & & \\
\hline Se aplicó cambio de pigmento. & & & \\
\hline \multicolumn{2}{|l|}{ ACTIVIDADES DEL OPERADOR QUE RECIBE EL TURNO } \\
\hline
\end{tabular}

\section{Si}

\begin{tabular}{|l|l|l|l|} 
Actividades & $\mathrm{Si}$ & No & Observaciones \\
\hline Se realizó de manera correcta el llenado de la & & &
\end{tabular}

Se realizó de manera correcta el llenado de la
bitácora de producción.

Se encuentra limpia y ordenada el área de

trabajo.

Se realizaron cambios de molde o de orden de

producción.

Se llenaron correctamente los formatos de

parámetros de moldeo.

\begin{tabular}{|l|l|l|l|} 
Se pesó la preforma y la plasta resultante de & & &
\end{tabular}

las distintas máquinas.

avisos de falla.

Se modificaron los parámetros de moldeo

durante el turno.

Se monitoreo cada una de las maquinas que

estuvieron trabajando.

Se inspeccionó que el pigmento no estuviera

contaminado.

Se inspeccionó que el pigmento líquido no

estuviera asentado en el fondo.

Se aplicó cambio de pigmento.

Nota: El operador que recibe el turno, en caso de no estar de acuerdo con algún punto que este marcado como "realizado" por el operador que

entrega el turno, hacerlo saber marcando la inconformidad en las

observaciones y comunicar al supervisor del turno.

Figura 5 Check List

Fuente: Elaboración propia con información de la empresa (2020) 


\section{Anexo 2}

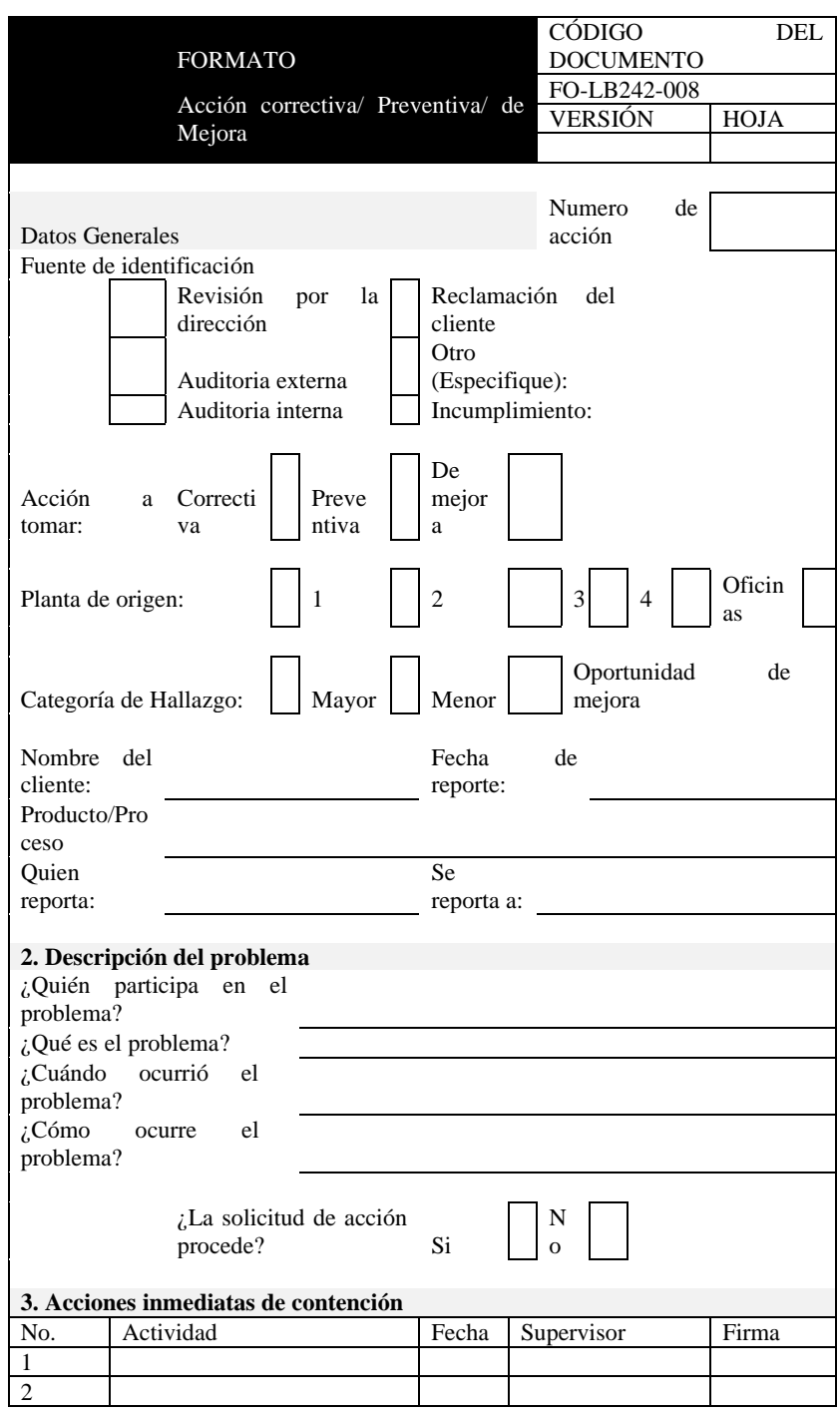

Figura 6 Control de acciones

Fuente: Elaboración propia con información de la empresa (2020)

\section{Agradecimiento}

Al Instituto Tecnológico Superior de Huauchinango y a la academia de Ingenieria Industrial por permitir el desarrollo de este trabajo de investigación.

\section{Conclusiones}

Con la implementación del ciclo DMAIC correspondiente a la metodología Six Sigma se generó un sistema de aseguramiento de la calidad (SAC) el cual tiene como objetivo principal la reducción de la variación de tono de las piezas terminadas, proceso que se efectúa en la zona de pigmentación del proceso de inyección-soplo de plástico.
El sistema de aseguramiento de la calidad originó un aumento de $0.3 \sigma$ con respecto al nivel de $3.4 \sigma$ registrado al inicio del proyecto el cual generaba un costo mensual por productos defectuosos de $\$ 24,442.50$, al final del proyecto se establece un nivel de $3.7 \sigma$ que deriva un costo mensual por productos defectuosos de $\$ 12,682.50$, la mejora antes mencionada trae consigo un beneficio económico mensual de $\$ 11,760.00$ representando un ahorro del $48 \%$ de los costos generados por variación de tono, proyectando un beneficio anual de $\$ 141,120.00$.

De igual forma se disminuyó en un $78 \%$ las quejas/inconformidades por parte de los clientes asegurando la aceptación futura de los pedidos fabricados.

La aplicación antes analizada confirma que la selección correcta de la metodología (DMAIC), y la aplicación de herramientas de Ingeniería traen consigo múltiples beneficios cuantitativos y cualitativos que aportan directamente al área de calidad y productividad de las organizaciones productivas, mejorando significativamente el nivel de satisfacción de los clientes.

\section{Referencias}

Albert, E. N., Soler, V. G., \& Molina, A. I. P. (2017). Metodología e implementación de Six Sigma.

Bikram Singh Kjit (2015). Wrap the scrap with DMAIC: Strategic Deployment of Six Sigma in Indian Foundry Smes. Anchor Academic Publishing.

Bookdown.org/asun_mayoral/book-16s/dmaic2medir.html

Duncan Acheson, J. (1996). Control de Calidad Y Estadística Industrial. Editorial Alfaomega. México.

Eckes, George. (2006). El Six Sigma para todos. Editorial Norma.

Fuchs C. \& Benjamini Y. (1994). Multivariate Profile Charts for Statiscal Process Control. Technometrics, 36 pp. 182 - 195. 
Lande, M., Shrivastava, R. L., \& Seth, D. (2016). Critical success factors for Lean Six Sigma in Smes (small and medium enterprises). The TQM Journal.

López, C. (2011) La metodología Seis Sigma ¿qué es? ¿Para qué sirve? ¿Cómo se aplica? ¿Requerimientos para su implementación? ¿Etapas de implementación? Recuperado de http://www.gestiopolis.com/recursos/experto/ca tsexp/ pagans/ger/no12/6sigma.html

López, G. (2001). Metodología Six-Sigma: calidad industrial. Artículo consultado en EBSCO, Baja California, México, Investigador del instituto de Ingeniería, UABC.

Pérez-López, E., \& García-Cerdas, M. (2014). Implementación de la metodología DMAICSeis Sigma en el envasado de licores en Fanal. Revista tecnología en Marcha, 27(3), ág88.

Peter S. Pande, R. P. (2000). The Six Sigma way. McGraw-Hill.

Rivera, L. N. M. (2006). Seis Sigma/Six Sigma: Guía Para Principiantes/Guide for Beginners. Panorama Editorial.

Socconini, Luis; Reato, Carlo. (2019) Lean Six Sigma. Sistema de gestión para liderar empresas. MARGE BOOKS.

Terrés-Speziale, A. M. (2007). SIX SIGMA: determinación de metas analíticas. Rev Mex Patol Clin, 54(1), 28-39.

Timans, W., Ahaus, K., van Solingen, R., Kumar, M., \& Antony, J. (2016). Implementation of continuous improvement based on Lean Six Sigma in small-and mediumsized enterprises. Total Quality Management \& Business Excellence, 27(3-4), 309-324.

Yang, C. C. (2004). Multivatiate statiscal methods and Six Sigma. Volumen uno. Número uno.

Zhang, M., Wang, W., Goh, T. N., \& He, Z. (2015). Aplicación completa de Six Sigma: un caso de estudio. Planificación y Control de Producción, 26(3), 219-234. 